\title{
Cephalometric Growth Analyses of the Human Upper Face Region During the Last Two Trimesters of Gestation ${ }^{1}$
}

\author{
ALPHONSE R. BURDI \\ Department of Anatomy, The University of Michigan, \\ Ann Arbor, Michigan 48104
}

\begin{abstract}
This study was designed to demonstrate quantitative growth trends in the human upper face region before birth. Photographs of 68 sagittally sectioned fetal heads were measured using a series of linear and angular measurements for changing height, length and shape. Cross-sectional types of data were treated with a statistical model which tested for linearity of the data, correlation between growth changes and increasing fetal age, significance of the differences between rates of change in related upper face regions and the general significance of the trends shown in the study.

Pooled data for the second and third trimesters suggested three distinct growth trends. Linear measurements of the cranial base, nasal area, and palate correlated significantly with increasing crown-rump length. Finally, a composite upper face profile for the sample suggested a relative migration of the region downward and forward away from the anterior cranial base and the hypophyseal fossa. These three trends collectively demonstrated that the pattern of upper facial growth before birth involves progressive enlargement of a relatively static profile. It should be understood that this pattern is a group trend as shown by cross-sectional sampling of many individuals over a period of time with no individual being measured more than once. Conversely, variabilities in a specific individual's growth are most appropriately demonstrated by measuring that individual several times throughout some time span, i.e., by longitudinal sampling. Studies of the human fetus are restricted generally to the cross-sectional sampling technique.

The close similarity of these prenatal trends with those reported for postnatal craniofacial growth suggests that certain patterns of facial growth in childhood can be seen as early as the beginning of the fetal period and emphasizes the continuum of human development.
\end{abstract}

Growth of the human face is a dynamic developmental continuum which begins in the embryo and continues throughout postnatal life. This continuum involves a timerelated interplay between differentiation at cellular, tissue and organ levels, the development of function, and morphogenic changes in size and shape.

The present study is specifically concerned with changes in size and shape of the human upper face before birth. Postnatal changes in size and shape of the face have received considerable attention from many workers representing a broad spectrum of scientific disciplines and techniques, as well as clinical interests. However, apart from the classic work of Scammon and Calkins ('29), few studies have been concerned

AM. J. ANAT., 125: 113-122. specifically with human prenatal cephalometries and general patterns of growth before birth. The reported studies of the human face before birth have been concerned more with descriptive, non-metric accounts of regional morphogenesis, the development of specific systems or structures and with providing embryological explanations for congenital defects and abnormal growth patterns of the postnatal face. Recently, greater emphasis has been directed toward filling the existing void of cephalometric data on size, form and directional growth in statistically representative

This investigation was supported, in part, by research grant HD 00178 from the National Institute of Child Health and Human Development, National Institutes of Health, USPHS. 
populations of human fetuses. In other words, "How fast and in what direction is the human face growing before birth?"

The purpose of this study is to demonstrate trends in changing height, length and shape of the human upper face before birth. Possible differences between second and third trimester growth patterns are also emphasized. Finally, to emphasize the continuum of human development, the observed prenatal patterns are related to growth trends reported for the upper face during the first years of postnatal life.

\section{MATERIALS AND METHODS}

Sixty-eight human fetuses representing ages 12 weeks through term or 70 through $420 \mathrm{~mm}$ crown-rump length (CRL) were selected for this study. This selection was made on the basis that all specimens were fixed in $10 \%$ neutral formalin and were free from gross craniofacial defects.
The procedures of specimen preparation, the definition of basic cephalometric landmarks, and the detailed descriptions of linear and angular measurements have been reported (Burdi, '65). In general, cephalometric analyses consisted of both linear and angular measurements based on such landmarks as nasion ( $N)$, sella $(\mathrm{S})$, basion (Ba), crista galli (CG), septal point (SP), anterior nasal spine (ANS), posterior nasal spine (PNS) and uvular point (UP). Linear measurements reflected changes in height and length of the cranial base, nasal septum, and palate. Changes in shape of these regions were analyzed by a series of angular measurements.

Cross-sectional type data derived from the study sample of 68 fetuses were analyzed using a statistical model designed and implemented in consultation with the staff of The University of Michigan Statistical Research Laboratory. Initially, an IBM

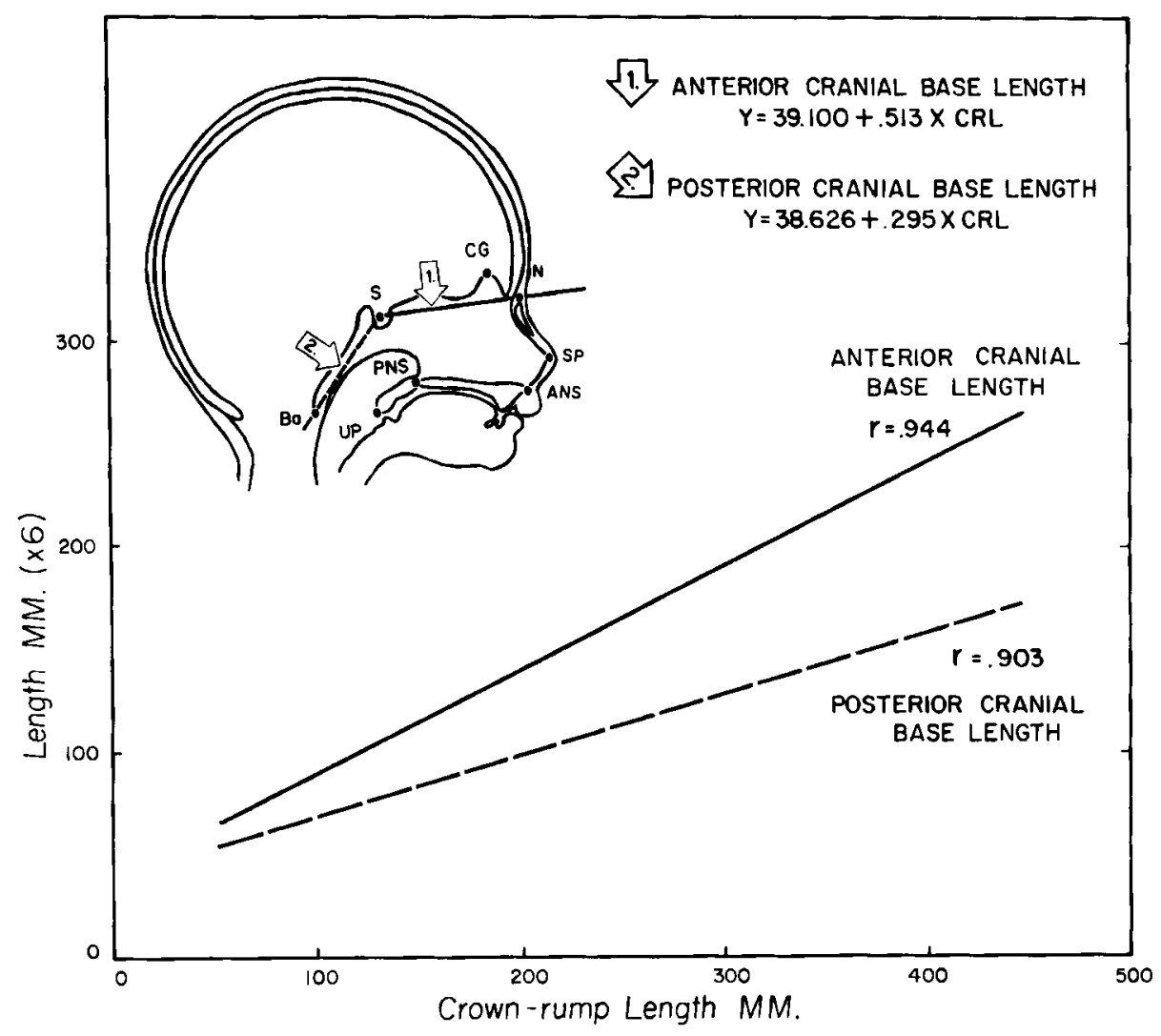

Fig. 1 Growth in length of the cranial base. 
7090 curve-fitting program was used to identify the mathematical function (linear, quadratic, or exponential) which best described the association between increasing age and distribution of the data. Once the best curve-fit was described as linear, according to the equation $\mathbf{y}=\mathbf{a}+\mathbf{b x}$, a regression line was drawn through the array of data points on the scattergram. In this equation, the value $a$ indicates where the regression line intercepts the $y$-axis. The regression coefficient $(b)$ describes the slope of the line or rate of change, i.e., the amount of change in a specific measurement per unit of change in fetal age (value $x)$. Correlation coefficients $(r)$ were developed simultaneously to demonstrate the intensity of the association between the two variables and to indicate the degree of accuracy with which the value of one variable can be estimated, given the value of the other. In a follow-up procedure, the significance of the difference between regional cephalometric growth trends was determined using the t-test. A final procedure was used to decide whether the growth trends observed in the study sample of 68 fetuses were representative of the theoretical population or universe from which the sample was drawn. Using regression and correlation coefficient values generated in the sample, upper and lower limits were computed between which population coefficient values can be expected to fall at a stated confidence level. For example, as in this study, if the limits are set at the $95 \%$ confidence level, it can be stated with $95 \%$ confidence that the population coefficients lie within the defined limits. Also, if the confidence interval does not include zero, it can be stated with $95 \%$ confidence that the population coefficient is not zero. This procedure provided a convenient basis for making estimates of facial growth patterns in the general population using data derived from the study sample of limited numbers.

\section{RESULTS}

General character of the grouth curves. Initial treatment of the data supported the

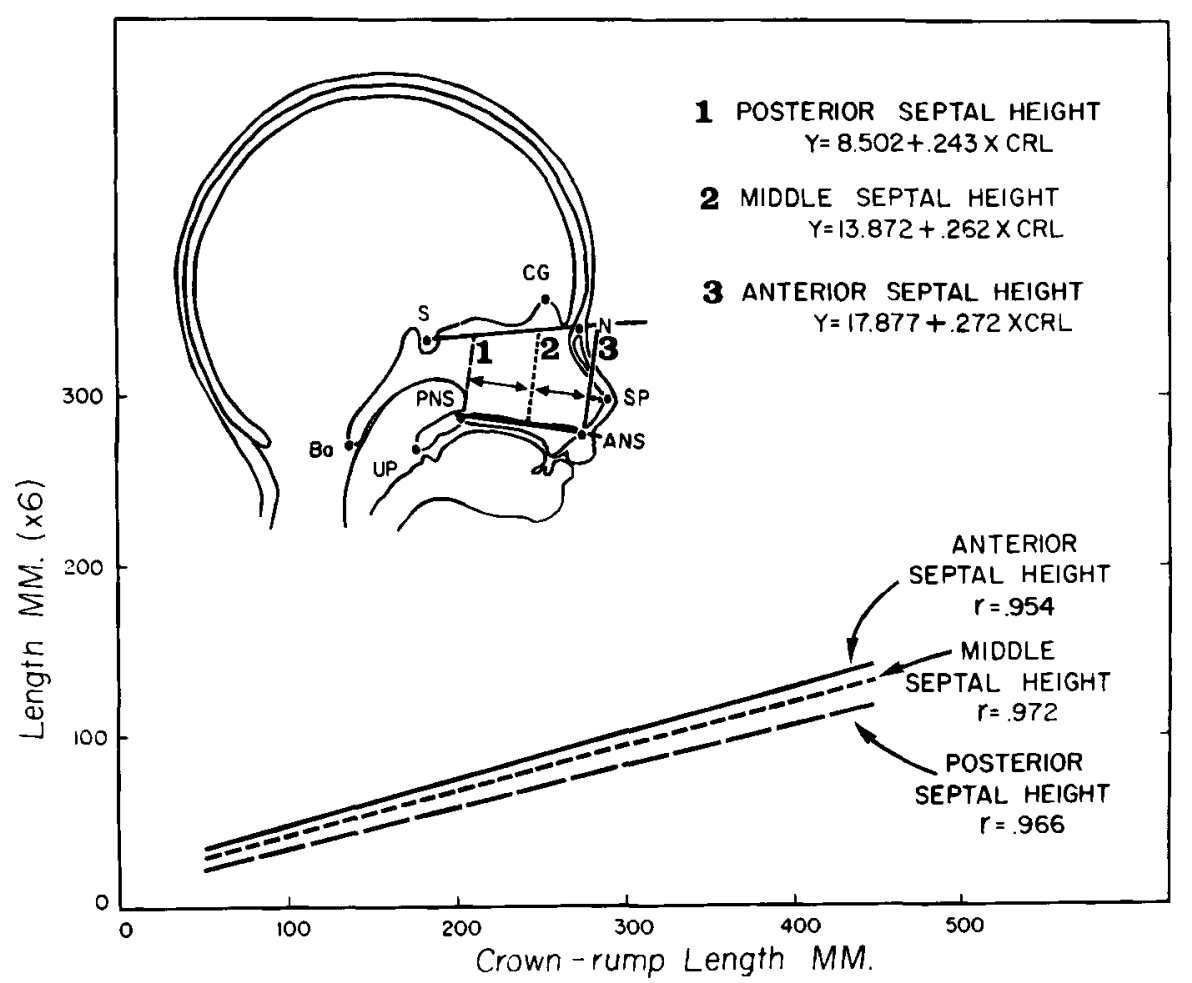

Fig. 2 Growth in height of the nasal septum. 


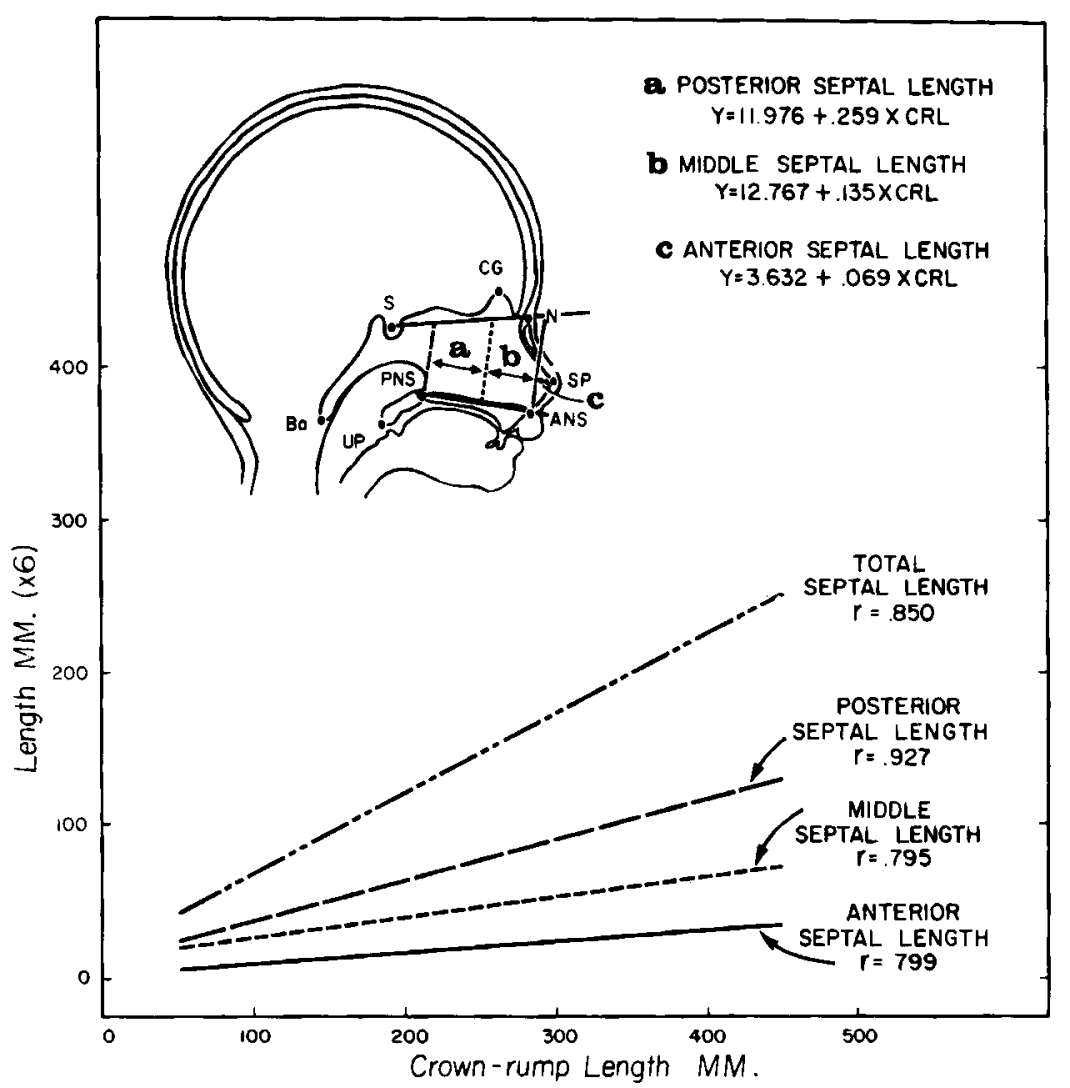

Fig. 3 Growth in length of the nasal septum.

basic hypothesis that the best fit of the observations (y-axis) might be significantly linear when related to measurements of crown-rump length ( $x$-axis). Simultaneous tests were performed also to show whether a significant departure from linearity, if shown, was haphazard or of sufficient regularity to suggest the presence of some type of systematic curvature. A comparison of these linear and curvilinear tests did not disprove the initial hypothesis of data linearity.

Cephalometric analyses of size. Increases in length of the cranial base were highly correlated with increases in fetal crown-rump length in the cross-sectional sample from 70 through $420 \mathrm{~mm}$ (fig. 1). As shown in table 1 , sample $r$ values for the anterior and posterior cranial base lengths, the total cranial base length and the length of the basicranial axis were significant $(p=0.05)$ when compared with upper and lower confidence limits of $\mathbf{r}$ values. A com- parison of the anterior and posterior cranial base lengths (table 2) indicated that the regression coefficient $(b=0.513)$ for the anterior cranial base was significantly greater than the regression coefficient $(b=$ 0.295 ) for the posterior base length. Throughout the series, the length dimensions of the anterior cranial base exceeded those of the posterior base. On a percentage basis, for example, the anterior cranial base consistently contributed more than one-half to the total base length, e.g., $55 \%$ at $70 \mathrm{~mm}$ CRL, $61 \%$ at $420 \mathrm{~mm}$ CRL.

Changes in each of the three regional septal height perpendicular lines correlated positively with increases in fetal crownrump length (fig. 2). Regression and correlation coefficients for the sample were well within the $95 \%$ confidence limits for $r$ and $b$ values (table 1 ). No significant differences were observed in the rates at which the anterior, middle and posterior septal heights regressed on increasing crown-rump 


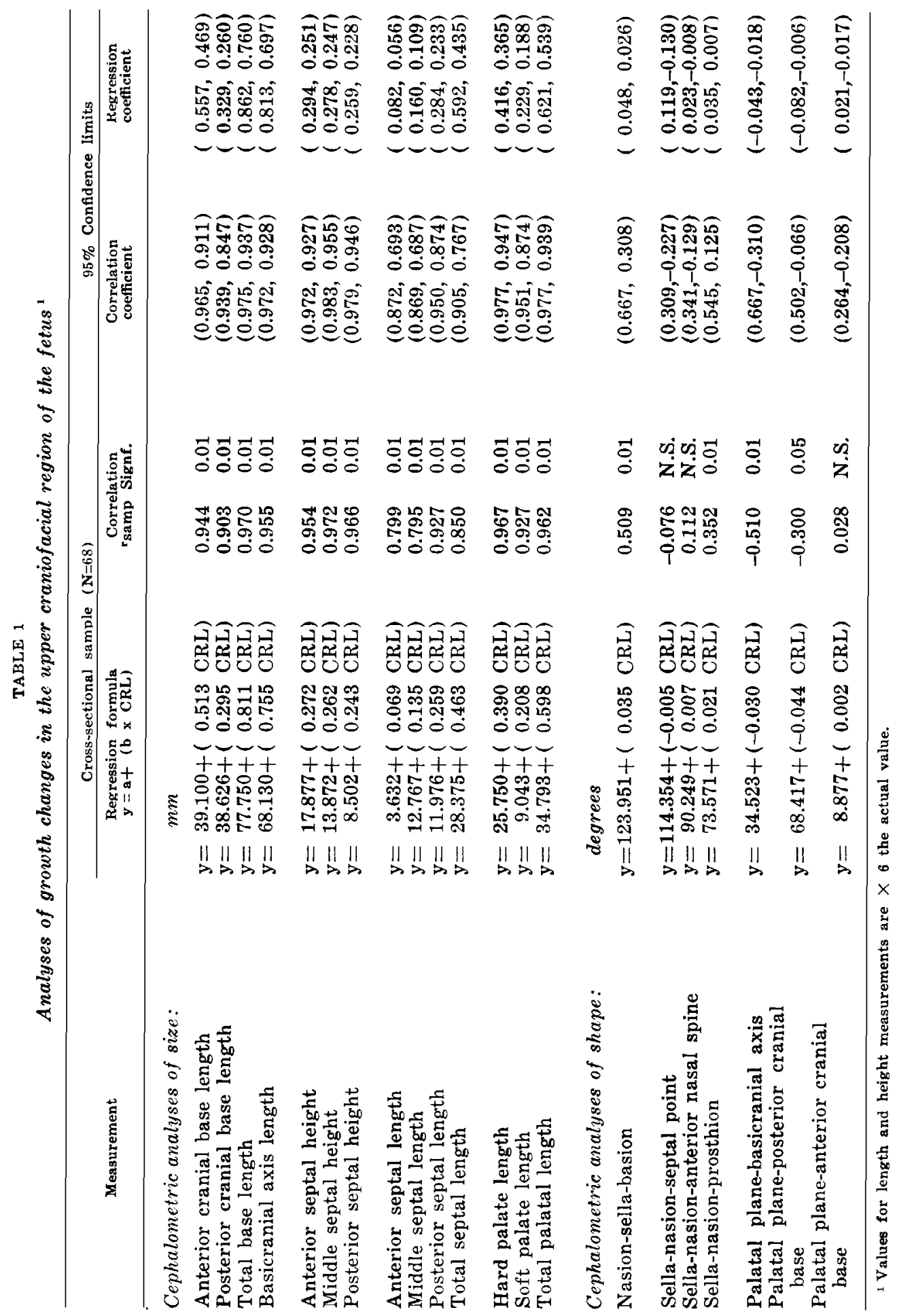


TABLE 2

Comparison of regression coefficients (b) of related craniofacial dimensions

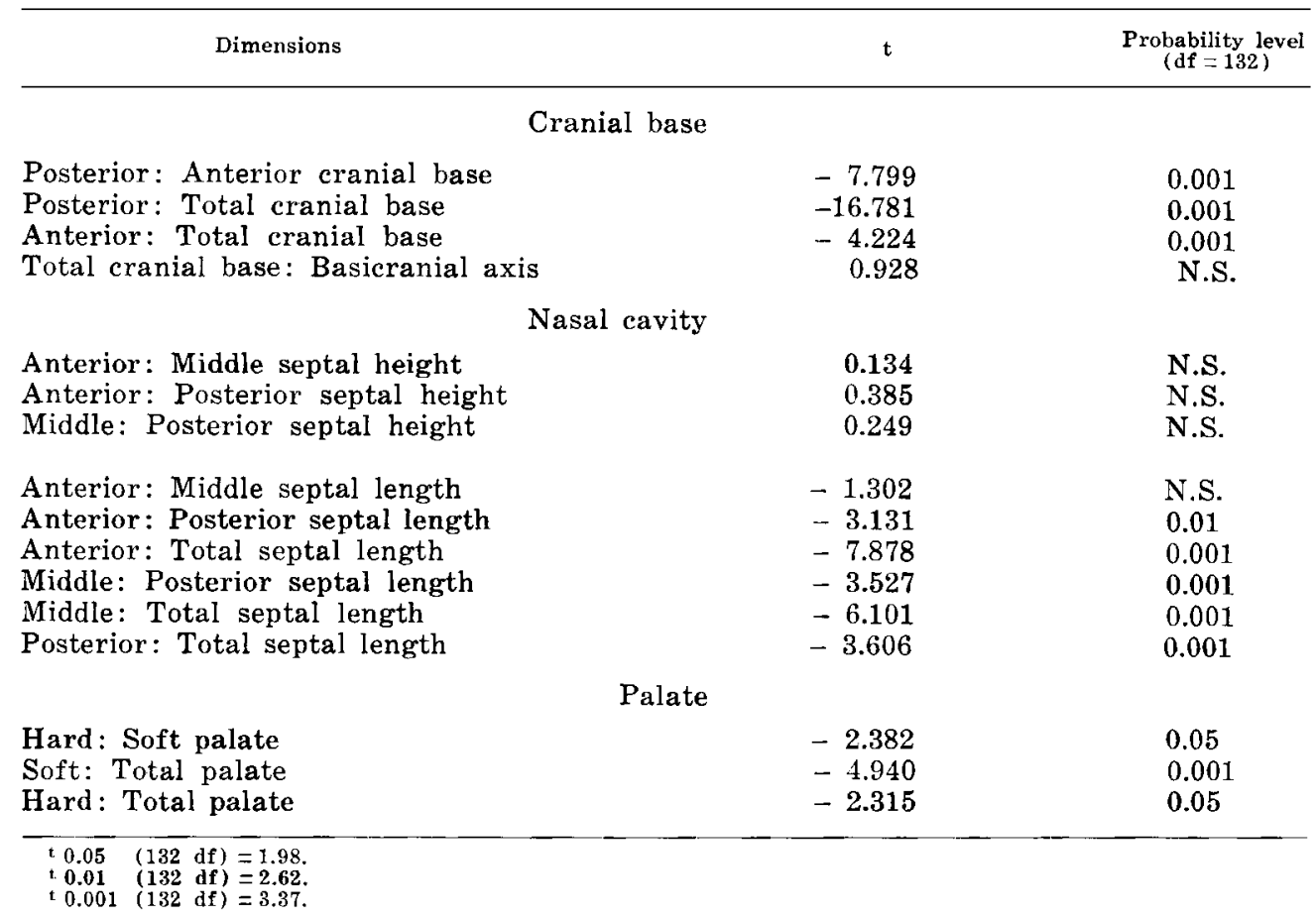

length (table 2). Similar high correlations were found between increases in septal length and changing fetal body length (fig. 3). Differences were observed, however, in the rates at which anterior, middle and posterior septal lengths regressed on increasing fetal length (table 2). With one exception, significant $t$-test values between regression coefficients for all regional septal length comparisons were observed. No significant difference was observed in the regression coefficients for anterior and middle septal lengths.

Increases in palatal length (fig. 4) were highly correlated with increasing crownrump length. Significant differences did occur between the regression coefficients for lengths of the hard and soft palate regions (table 2). On a relative basis, the length of the hard palate consistently contributed approximately $65 \%$ to the total palatal length.

Cephalometric analyses of shape. Only a moderate correlation was observed between changes in the angular relationship between anterior and posterior cranial base segments when related to increasing crownrump length (fig. 5, table 1). Although markedly lower than regression and correlation coefficient values for the changes in cranial base lengths, the regression coefficient for the angle N-S-Ba $(b=0.035)$ as well as its correlation coefficient $(r=$ 0.509 ) were significant ( $p=0.05$ ).

No significant changes in regression coefficients were observed for the upper facial profile or shape of the external nose. The general pattern of profile stability was similarly supported by low values for the regression and correlation coefficients of the three major facial angles (table 1).

Angular relationships between the palatal plane and the two cranial base segments were correlated, although negatively, with increases in crown-rump length (fig. 6). Table 1 indicates a closing of the angles between the palatal plane and posterior cranial base and between the basicranial axis. No significant change in trends was observed in the relationship between the palatal plane and the plane of the anterior cranial base. 


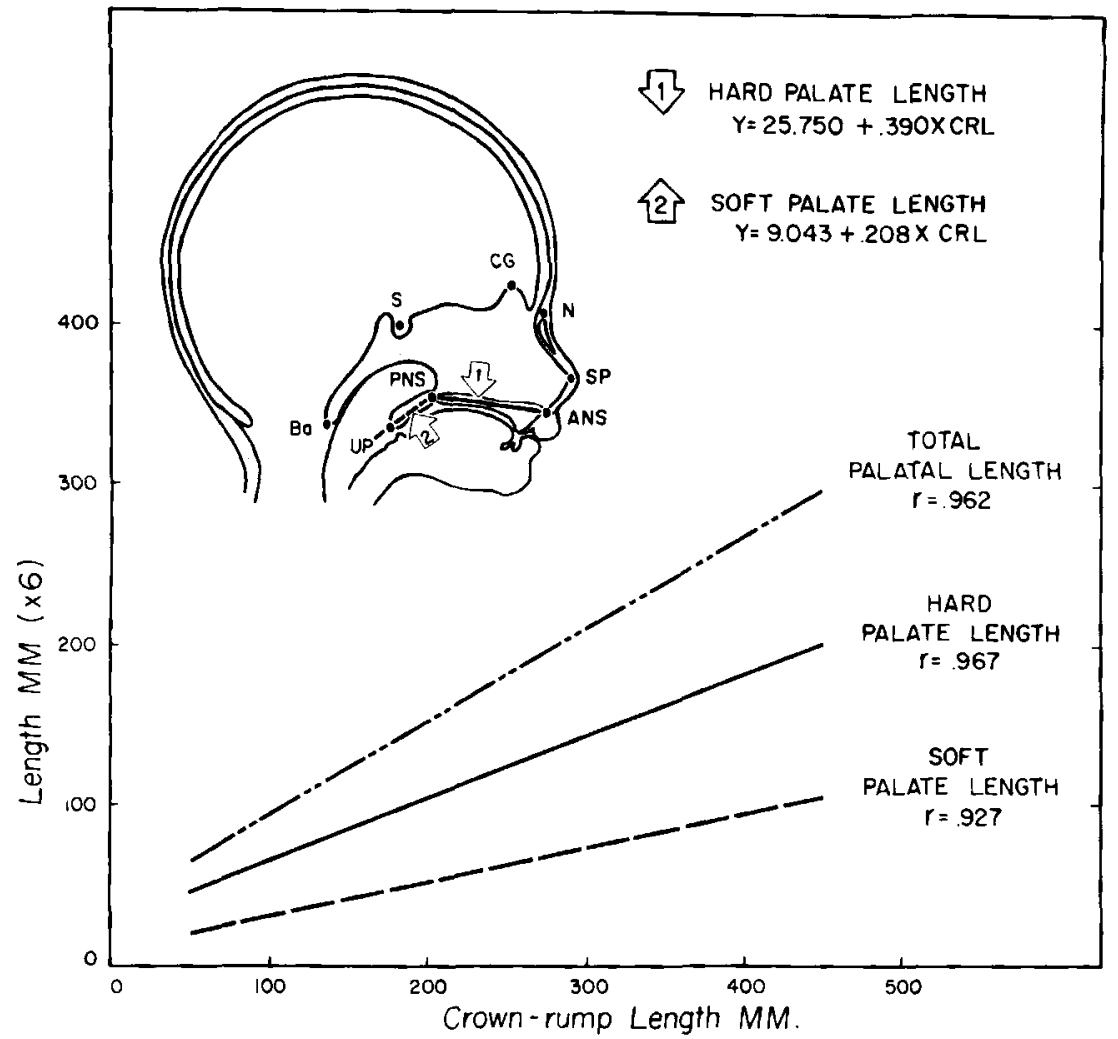

Fig. 4 Growth in length of the palate.

\section{DISCUSSION}

The major finding of this study was that the growth of the human upper face during the last two trimesters in utero followed a trend characterized by progressive enlargement of a relatively stable profile. This observation extends into the third trimester a growth trend which was reported earlier (Burdi, '65) for a cross-sectional sample of second trimester fetuses. In that study of dissected fetuses, increases in both height and length of the upper face region were directly and positively correlated with increases in fetal age or crown-rump length. Angular or shape changes, on the other hand, did not show this positive linear relationship with changes in crown-rump length. In addition, the profile of the upper face appeared to shift downward and forward away from the anterior cranial base and the pituitary fossa. The present study of pooled data from the second and third trimesters supports an observation (Ford, '56) that the proportions of the nasal area are relatively constant throughout the fetal period as the area shows progressive increases in size. It was also demonstrated that the fetal head, as a whole, underwent some change in shape as the collective result of differential growth rates in its several component regions.

The general pattern of increasing facial size while maintaining a relatively constant shape can also be supported by studies which have used specimen preparations and analytic techniques that differ from those used by either Ford or Burdi. Inoue ('61) employed roentgenographic cephalometry in a growth study of 242 undissected fetal heads. Statistical analyses of his x-ray films also showed a pattern of increasing facial size with no marked change in facial profile. No curvilinearity of the height-length cephalometric data was reported. Recently, Levihn ('67) supplemented Inoue's roentgenographic study and likewise found a relative constancy of facial shape. Levihn did indicate, however, a curvilinear or sig- 


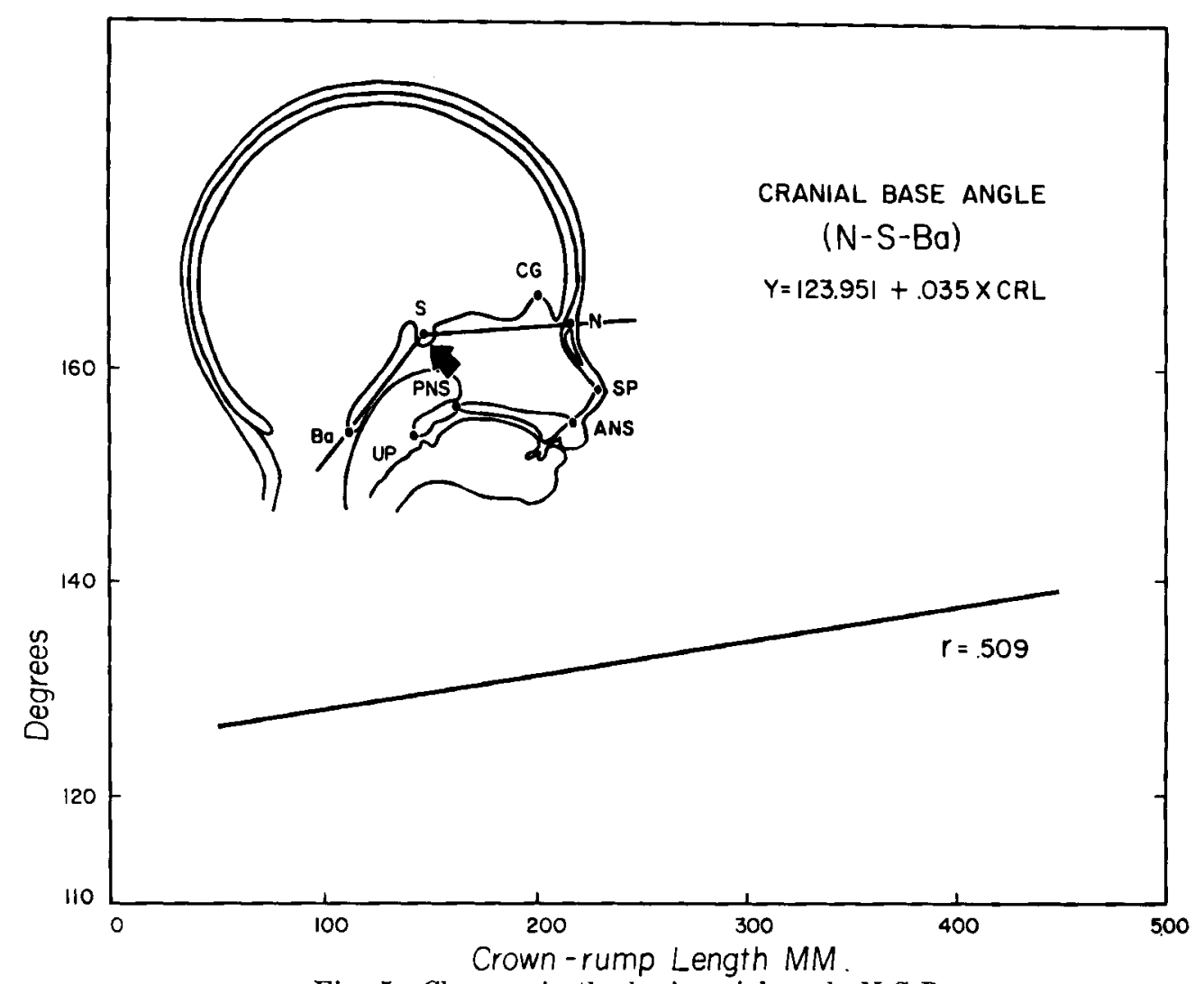

Fig. 5 Changes in the basicranial angle N-S-Ba.

moid distribution of data for non-angular measurements. Based on group means and derived percentages without statistical curve fitting procedures, it was shown that the fastest growth rates occurred during the fourth and fifth lunar months with a gradual plateauing thereafter.

The present study of second and third trimester fetuses showed a moderate increase of about $12^{\circ}$ in the angle between the anterior and posterior cranial base segments. This increase was not evident when second trimester data was inspected separately. Yet, this moderate increase in the basicranial angle is consistent with the findings of Ford ('56). When projected to the perinatal period, this prenatal angular relationship also shows a clear continuity with the degree of basal flexure reported in the orthodontic literature (Bjork, '55; Brodie, Jr., '55; Stramrud, '59; Koski, '60 ; McNeill, '62).
That a linear and not a curvilinear relationship may be operational for the fetal face is supported by the high positive correlations between increasing crown-rump length and increasing height-length parameters. This demonstration of a linear growth pattern of the prenatal upper face provides a quantitative refinement of classical descriptions which broadly characterize prenatal body growth as following a sigmoid curve, with its highest peak of activity occurring during the second trimester (Toldt, 1879; Mall, '10 ; Zangemeister, '11; Streeter, '20).

Observations of this study support the concept of a developmental continuum between pre- and postnatal facial growth. Broadbent ('37) indicated that the basic shape of the child's face is first recognizable at the time of full eruption of the deciduous teeth. Brodie ('41) argued for a relative constancy of cross-sectional facial profiles 


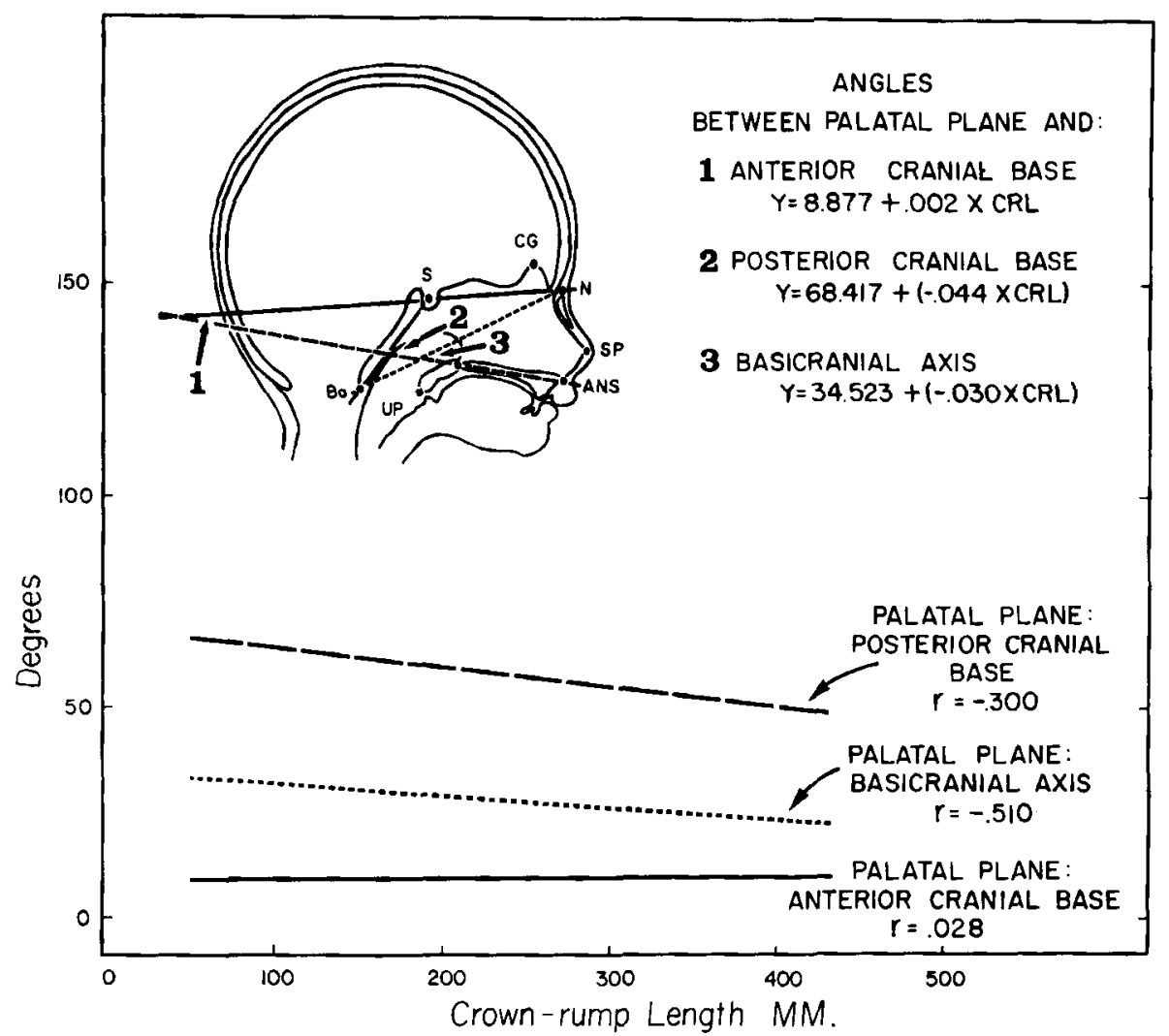

Fig. 6 Angular relations between the palatal plane and select basicranial planes.

which appears as early as the third postnatal month. Ortiz and Brodie ('49) later found that the geometric profile of the human face can be recognized as early as birth. The present study, as a sequel to these postnatal observations, lends support to the idea that the young human face possesses a basic shape. It further suggests that the postnatal pattern of enlarging facial profiles can be recognized as early as the second trimester before birth.

\section{ACKNOWLEDGMENT}

The author would like to acknowledge Esther B. Schaeffer of the Statistical Research Laboratory, The University of Michigan, for her advice on the design of the statistical model and the subsequent analyses of the growth data.

\section{LITERATURE CITED}

Bjork, A. 1955 Cranial base development. Amer. J. Orthodont., 41: 198-226.
Broadbent, B. H. 1937 The face of the normal child. Angle Orthodont., 7 : 183-207.

Brodie, A. G. 1941 On the growth pattern of the human head from the third month to the eighth year of life. Am. J. Anat., 68: 209-262.

Brodie, A. G., Jr. 1955 The behavior of the cranial base and its components as revealed by serial cephalometric roentgenograms. Angle Orthodont., 25: 148-160.

Burdi, A. R. 1965 Sagittal growth of the nasomaxillary complex during the second trimester of human prenatal development. $J$. Dent. Res., 44: 112-125.

Inoue, N. 1961 A study on the developmental changes of dentofacial complex during fetal period by means of roentgenographic cephalometrics. Bull. Tokyo Med. and Dent. Univ., 8: 205-227.

Koski, K. 1960 Some aspects of the growth of the cranial base and the upper face. Sart. ur Odont. Tskr., 68: 344-358.

Levihn, W. C. 1967 A cephalometric roentgenographic cross-sectional study of two craniofacial complexes in fetuses from 12 
weeks to birth. Am. J. Orthodont., 53: 822848.

Mall, F. P. 1910 Determination of the age of human embryos and fetuses. In: Human Embryology. F. Keibel and F. P. Mall, eds. J. P. Lippincott Co., Philadelphia. Vol. 1, Chap. VIII, pp. 180-201.

McNeill, R. W. 1962 A roentgenographic cephalometric study of nasopharyngeal and cranial base growth in cleft palate children. Thesis (M.S.), University of Pennsylvania, Philadelphia.

Ortiz, M. H., and A. G. Brodie 1949 On the growth of the human head from birth to the third month of life. Anat. Rec., 10\%: 311333.

Scammon, R. E., and L. A. Calkins 1929 The Development and Growth of the Ex- ternal Dimensions of the Human Body in the Fetal Period. The University of Minn. Press, Minneapolis.

Stramrud, L. 1959 External and internal cranial base. A cross-sectional study of growth and of association in form. Acta Odont. Scand., 17: 239-266.

Streeter, G. L. 1920 Weight, sitting height, head size, foot length and menstrual age of the human embryo. Contrib. to Embryology, Carnegie Institute, 11 : 143-179.

Toldt, C. 1879 Uber die Altersbestimmung menschlicher Embryonen. Prager med. Wochenschr., 4: 121-140.

Zangemeister, W. 1911 Die Altersbestimmung des Foetus nach graphischer Methode. Ztschr. f. Geburtsh. u Gynakol., 69: 127142 . 